\title{
DESCRIPCIÓN DE PACIENTES CON TAMIZAJE POSITIVO PARA HIPERTENSIÓN PULMONAR TROMBOEMBÓLICA CRÓNICA.
}

\author{
Daniela Cerón Perdomo1, Daniela Patino Hernández1, Pablo Gil Torres2, Claudio Villaquirán Torres2. \\ Departamento de medicina Interna, Hospital Universitario San Ignacio Bogotá - Colombia. \\ Unidad de neumología Departamento de medicina Interna, Hospital Universitario San Ignacio Bogotá- Colombia
}

\section{OBJETIVOS}

La hipertensión pulmonar tromboembólica crónica (HPTEC) es una complicación infrecuente, asociada con una alta tasa de morbimortalidad. Actualmente no existe una guía para la búsqueda activa de pacientes asintomáticos. Sin embargo, se han desarrollado puntajes de predicción de riesgo que pueden ser una herramienta útil en la identificación de pacientes con alto riesgo de desarrollar HPTEC.

\section{MÉTODOS}

Se realizó la búsqueda en el registro RETEP-HUSI que cuenta con los pacientes diagnosticados con tromboembolismo pulmonar en el Hospital Universitarios San Ignacio entre los años 2017 - 2018. Se realizó en todos los pacientes el cálculo de la escala de predicción de riesgo de HPTEC propuesto por Klok y cols. Para los pacientes con escala positiva se realizó una descripción clínica y demográfica.

\section{REFERENCIAS}

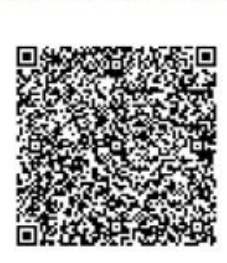

\section{RESULTADOS}

De los 113 pacientes del registro RETEP-HUSI, 9.7\% presentaron una escala de riesgo positiva. De estos el $72.7 \%$ fueron mujeres, con una edad media de 61.7 años. El principal factor de riesgo fue presentar antecedente de trombosis venosa profunda $36.4 \%$ y antecedente de TEP 9.9\%. Los principales síntomas de ingreso fueron disnea

$100 \%$, dolor torácico $54.6 \%$ y hemoptisis $18.2 \%$. La escala PESI estableció un riesgo muy alto en el $54.6 \%$ de los pacientes y el $54.5 \%$ de los pacientes presentaron disfunción ventricular derecha.

\section{CONCLUSIÓN}

Un bajo porcentaje de pacientes presenta escala de riesgo positiva para HPTEC. En los pacientes con estas características se debe realizar una búsqueda activa con el fin de identificarlos tempranamente $\mathrm{y}$ proporcionarles un tratamiento adecuado. Futuros estudios son necesarios para evaluar los desenlaces de pacientes que presentan escala positiva.
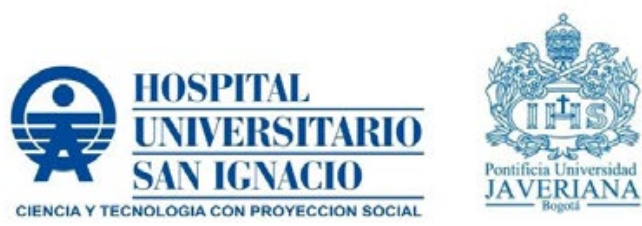

Revista Colombiana de Neumología Vol. 31 N. ${ }^{\circ} 2 \mid 2019$ 